\title{
Opinion Formation in Laggard Societies
}

\author{
Peter Klimek ${ }^{1}$, Renaud Lambiotte ${ }^{2}$, Stefan Thurner ${ }^{1,2}$ * \\ ${ }^{1}$ Complex Systems Research Group; HNO; Medical University of Vienna; Währinger Gürtel 18-20; A-1090; Austria \\ ${ }^{2}$ GRAPES; Université de Liège; Sart-Tilman; B-4000 Liège; Belgium \\ 3 Santa Fe Institute; 1399 Hyde Park Road; Santa Fe; NM 87501; USA
}

\begin{abstract}
We introduce a statistical physics model for opinion dynamics on random networks where agents adopt the opinion held by the majority of their direct neighbors only if the fraction of these neighbors exceeds a certain threshold, $p_{u}$. We find a transition from total final consensus to a mixed phase where opinions coexist amongst the agents. The relevant parameters are the relative sizes in the initial opinion distribution within the population and the connectivity of the underlying network. As the order parameter we define the asymptotic state of opinions. In the phase diagram we find regions of total consensus and a mixed phase. As the 'laggard parameter' $p_{u}$ increases the regions of consensus shrink. In addition we introduce rewiring of the underlying network during the opinion formation process and discuss the resulting consequences in the phase diagram.
\end{abstract}

PACS numbers: 89.75.Fb, 87.23.Ge, 05.90.+m

\section{INTRODUCTION}

Many decisions of human beings are often strongly influenced by their social surroundings, e.g. the opinion of friends, colleagues or the neighborhood. Only a few types of decisions in few individuals emerge from absolute norms and firm convictions which are independent of the opinion of others. Much more common is the situation where some sort of social pressure leads individuals to conform to a group, and take decisions which minimize conflict within their nearest neighborhood. For example, if a large fraction of my friends votes for one party, this is likely to influence my opinion on whom to vote for; if I observe my peers realizing huge profits by investing in some stock this might have an influence on my portfolio as well; and if the fraction of physicist friends (coauthors) publishing papers on networks exceeds a certain threshold, I will have to reconsider and do the same; the social pressure would otherwise be just unbearable. Lately, the study of opinion formation within societies has become an issue of more quantitative scientific interest. In first attempts agents were considered as sites on a lattice, and opinion dynamics was incorporated by the so-called voter model (VM) [1] (only two neighbors influence each other at one timestep), the majority rule (MR) 2, 3] (each member of a group of odd size adopts the state of the local majority), or the Axelrod model [4] (where two neighbors influence themselves on possibly more than one topic with the objective to become more similar in their sets of opinions). Imposing regular lattice structure on social environments is convenient however, most observed structures of real-world networks belong to one of three classes: Erdös-Renyi (ER) [5], scale-free [6] or small-world networks [7]. This has been accounted for the VM 8, 9, 10] as well as for the MR on different topologies 11, 12, 13]. For a review of further efforts in this directions see [14, 15] and citations therein. Another

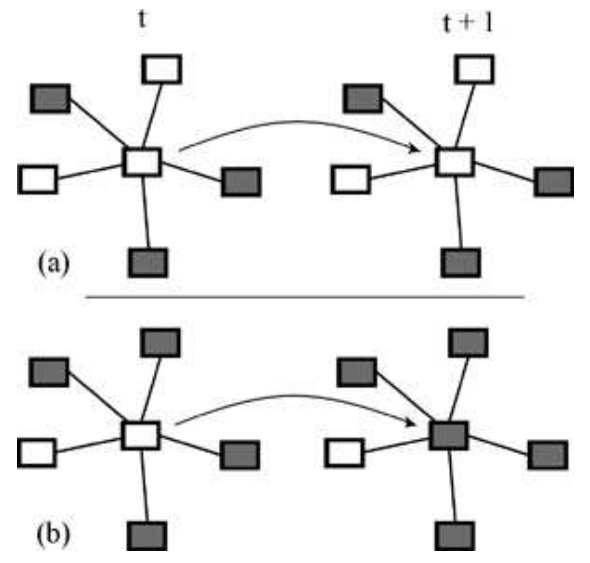

FIG. 1: Update process for two different configurations of neighbors and an update threshold of $p_{u}=0.8$. The node in the center gets updated. (a) Three out of five neighbors are in a different state, so the threshold is not exceeded and the node stays unchanged. (b) Four out of five neighbors are in a different state; $4 / 5 \geq 0.8$ thus the node adopts the state.

approach to model social interaction was developed out of the notion of catalytic sets [16], leading to an unanimity rule (UR) model [17] on arbitrary networks in an irreversible formulation.

As a realistic model for many real world situations here we present a reversible generalization to the UR and MR models introducing an arbitrary threshold governing updates ('laggard' parameter). The UR and MR are extremal cases of the model. In [18] the idea of a threshold was introduced in the context of investigating the origin of global cascades in ER networks of 'early-adopters'. In contrast to this work, where updates were only allowed in one direction, i.e. irreversible, the following model is fully reversible in the sense that two opinions compete against each other in a fully symmetric way. 


\section{THE MODEL}

Each individual $i$ is represented as a node in a network. The state of the node represents its opinion on some subject. For simplicity we restrict ourselves on binary opinions, yes/no, 0/1, Bush/Mother Theresa, etc. Linked nodes are in contact with each other, i.e. they 'see' or know each others opinion. The opinion formation process of node $i$ is a three-step process (see Fig 1): Suppose $i$ is initially in state ' 0 '(' 1 ').

- Check the state of all nodes connected to $i$.

- If the fraction of state ' 1 '(' 0 ')-nodes of $i$ 's neighbors exceeds a threshold $p_{u}, i$ adopts opinion '1'('0').

- Otherwise $i$ remains in state '0'('1').

As a substrate network we chose random graphs [5], i.e. $N$ nodes are randomly linked with $L$ links (selfinteractions are forbidden), the average connectivity being $\bar{k}=L / N$. The update threshold necessary for a node's change of opinion, $p_{u}$ has to be higher than 0.5 in order to be meaningful in the above sense. The update is carried out asynchronously. In a network containing $N$ nodes, at time $t$, there are $A_{t}^{0}$ nodes with opinion ' 0 ' and $A_{t}^{1}$ nodes with opinion '1'. The relative number of nodes are $a_{t}^{0 / 1}=A_{t}^{0 / 1} / N$. One time step is associated with applying the update procedure $N$ times, i.e. each node gets updated once per timestep on average. As time goes to infinity, the relative population of nodes with opinion $0 / 1$ will be denoted by $a_{\infty}^{0 / 1}$.

\section{ANALYTICAL AND NUMERICAL RESULTS}

To derive a master equation for the evolution of this system we calculate opinion-transition probabilities via combinatorial considerations in an iterative fashion, motivated by [16]. A master equation for $a_{t}^{0}$ is found explicitly, the situation for $a_{t}^{1}$ is completely analogous. At $t=0$, we have a fraction of $a_{0}^{0}$ nodes in state ' 0 '. The probability that at time $t$ one node belonging to $a_{t}^{0}$ will flip its opinion to ' 1 ' is denoted by $p_{t}^{0 \rightarrow 1}$. This probability is nothing but the sum over all combinations where more than a fraction of $p_{u}$ of the neighbors are in state ' 1 ', weighted by the probabilities for the neighboring nodes to be either from $a_{t}^{0}$ or $a_{t}^{1}=\left(1-a_{t}^{0}\right)$,

$$
p_{t}^{0 \rightarrow 1}=\sum_{i=\left\lceil\bar{k} p_{u}\right\rceil}^{\bar{k}}\left(\begin{array}{c}
\bar{k} \\
i
\end{array}\right)\left(1-a_{t}^{0}\right)^{i}\left(a_{t}^{0}\right)^{\bar{k}-i},
$$

where $\lceil$.$\rceil denotes the ceiling function, i.e. the near-$ est integer being greater or equal. The same consideration leads to an expression for the opposite transition $p_{t}^{1 \rightarrow 0}$, where 1 and 0 are exchanged in Eq.(1).
The probability for a node to be switched from ' 0 ' to ' 1 ', $\Delta_{0}^{0 \rightarrow 1}$, is the product of the transition probability, $p_{t}^{0 \rightarrow 1}$, and the probability to be originally in the fraction $a_{0}^{0}$, i.e. $\Delta_{0}^{0 \rightarrow 1}=p_{0}^{0 \rightarrow 1} a_{0}^{0}$. The same reasoning gives $\Delta_{0}^{1 \rightarrow 0}=p_{0}^{1 \rightarrow 0}\left(1-a_{0}^{0}\right)$ and provides the master equation for the first time step (i.e. updating each node once on average),

$$
a_{1}^{0}=a_{0}^{0}+\Delta_{0}^{1 \rightarrow 0}-\Delta_{0}^{0 \rightarrow 1} .
$$

Let us now examine some special cases.

\section{The low connectivity limit}

For sufficiently low connectivities $\bar{k}$ there are no more possible updates in the network after the first iteration. For a given update threshold $p_{u}$, this is the case if a change in opinion requires all neighboring nodes to have the same state. To see this more clearly, consider the case of a network with constant $k=2$ (1D circle). Choose a node whose state is e.g. ' 0 '. There are four possible configurations of neighbors: both being in state ' 0 ', one being in ' 0 ' and the other in ' 1 ' and both being in ' 1 '. Irrespective of $p_{u}$, only the latter configuration allows an update. For all other cases at least one neighbor in state ' 0 ' must be updated to '1', i.e. has to have two neighboring nodes in ' 1 '. But this is not possible, since at least one neighbor will always be in ' 0 '. The same holds for higher values of $\bar{k}$, as long as every neighbor has to hold the same opinion to allow an update, i.e. we effectively use an unanimity rule. For the special case $k=2$ the final population in state ' 0 ' is given by $a_{\infty}^{0}=a_{1}^{0}$. Inserting this in Eq.(2) yields

$$
a_{\infty}^{0}=3\left(a_{0}^{0}\right)^{2}-2\left(a_{0}^{0}\right)^{3} .
$$

A comparison between the theoretical prediction of Eq.(3) and the simulation of this system (on a regular 1D circle network with $N=10^{4}$ ) is seen in Fig[2(a).

\section{Higher connectivities}

For higher connectivities there are much more configurations allowing for potential updates, the evolution does not stop after one single iteration. At the second time step the update probability is given by the product of the transition probability at $t=1$ and the probability to be initially in the respective state, reduced by the probability to already have undergone this transition during the first time step. We have, for example, $\Delta_{1}^{1 \rightarrow 0}=\left(p_{1}^{1 \rightarrow 0}-p_{0}^{1 \rightarrow 0}\right)\left(1-a_{0}^{0}\right)$. For arbitrary times $t$ this is straight forwardly seen to be $\Delta_{t}^{1 \rightarrow 0}=$ $\left(p_{t}^{1 \rightarrow 0}-p_{t-1}^{1 \rightarrow 0}\right)\left(1-a_{0}^{0}\right)$, and the master equation becomes $a_{t+1}^{0}=a_{t}^{0}+\Delta_{t}^{1 \rightarrow 0}-\Delta_{t}^{0 \rightarrow 1}$. Inserting for $a_{t}^{0}$ in a recursive way yields the master equation

$$
a_{t+1}^{0}=a_{0}^{0}+p_{t}^{1 \rightarrow 0}\left(1-a_{0}^{0}\right)-p_{t}^{0 \rightarrow 1} a_{0}^{0} .
$$



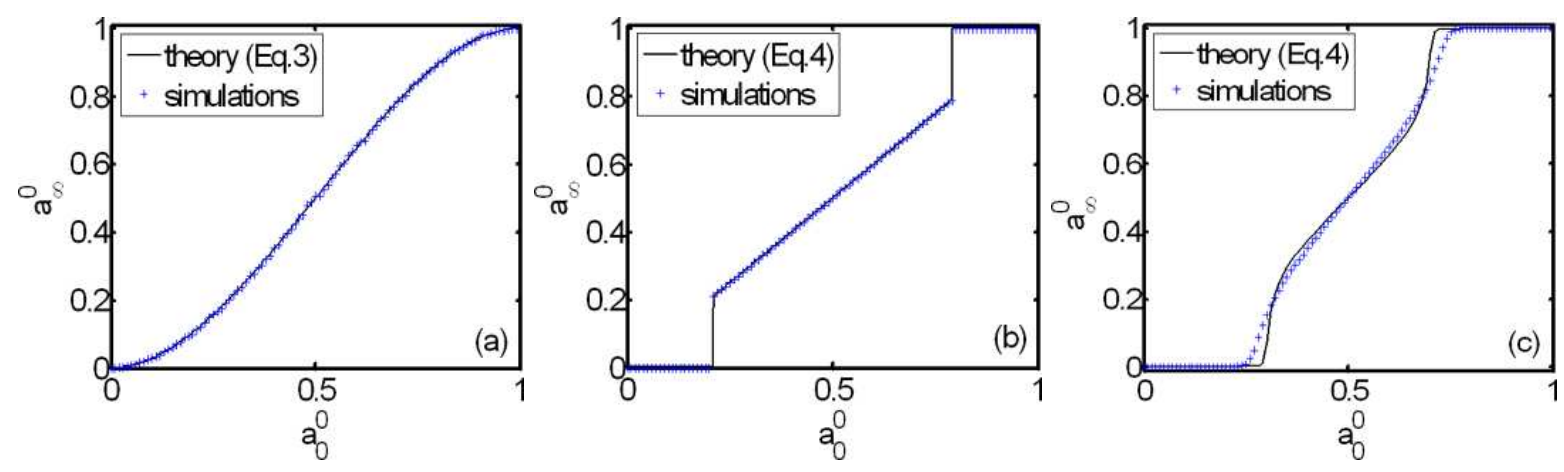

FIG. 2: Asymptotic population sizes of the ' 0 '-state fraction, $a_{\infty}^{0}$, as a function of its initial size, $a_{0}^{0}$, for $N=10^{4}, p_{u}=0.8$. (a) $k=2$ for all nodes (1D circle), (b) ER graph with $\bar{k}=9000$ and (c) ER graph with $\bar{k}=10$.

Again, theoretical predictions of Eq.(4) agree perfectly with numerical findings, Fig 2(b). Three regimes can be distinguished: two of them correspond to a network in full consensus. Between these there is a mixed phase where no consensus can be reached.

High connectivity limit. For the fully connected network the asymptotic population sizes can easily be derived: if $a_{0}^{0}>p_{u}$ or $a_{0}^{0}<1-p_{u}$ consensus is reached. For $1-p_{u}<a_{0}^{0}<p_{u}$ the system is frustrated and no update will take place, giving rise to a diagram like Fig 2 (b). Compared to Fig 2(a) a sharp transition between the consensus phases and the mixed phase has appeared. We now try to understand the origin of this transition.

Intermediate regime. The transition between the smooth solution for the final populations as a function of $a_{0}^{0}$ and the sharp one for higher connectivities becomes discontinuous when the possibility for an individual node to get updated in a later timestep ceases to play a negligible role. Systems with small update probabilities will then be driven towards the consensus states. However, if the initial populations are too far from the consensus states they will not be reached. For $p_{u}=0.8$ the sharp transition arises for values of $\bar{k}$ around 10. Fig 2(c) shows simulation data for ER graphs with $N=10^{4}$ nodes and $\bar{k}=10$ with $p_{u}=0.8$. Here we already find two regimes with consensus and an almost linear regime in-between. The analytical curve obtained from numerical summations of Eq. (4) resembles the qualitative behavior of the simulations up to finite-size deviations. The dynamics of the system is shown in the phase diagram, Fig[3(a). It illustrates the size of the respective regimes and their dependence on the parameters $a_{0}^{0}$ and connectedness $\bar{k} / N$. The order parameter is $a_{\infty}^{0}$. Along the dotted lines a smooth transition takes place, solid lines indicate discontinuous transitions from the consensus phase to the mixed phase. The change from smooth to sharp appears at $\bar{k} / N \approx 0.01$. For larger $p_{u}$ the regions of consensus shrink toward the left and right margins of the figure.

So far we assumed static networks. However, this is far from being realistic, as social ties fluctuate. We thus allow links to get randomly rewired with the rewirement process taking place on a larger time scale than the opinion update, since otherwise the new connection would not lead to state changes. Let us assume that the number of rewired links per rewirement-timestep is fixed to $L^{\prime}$, so that it becomes natural to define a social temperature, $T=L^{\prime} / L . T$ quantifies the individual's urge to reconsider a topic with new acquaintances, or equivalently, the fluctuation of ties in their social surrounding.

The evolution of opinions in a network at $T \neq 0$ is as follows: We fix a network and perform the same dynamics as for $T=0$, until the system has converged and no further updates occur. Then perturb the system by a rewirement step and randomly rewire $L^{\prime}$ links among the $N$ nodes ( $N$ and $L$ are kept constant over time), increase the time-unit for the rewirement steps by one and let the system relax into a (converged) opinion configuration. Iterate this procedure. Note that this process can be viewed as a dynamical map of the curves shown in Figs/2(a)-(c). With this view it becomes intuitively clear that consensus will be reached for a wider range of parameters, where the time to arrive there crucially depends on the value of $\bar{k}$.

To incorporate the temperature effect in the master equation we introduce the second timescale and denote the population in state ' 0 ' as $a_{t, \bar{t}}^{0}$. Here $t$ is the time for the update process as before and $\bar{t}$ is the time step on the temperature time scale, i.e. counts the number of rewirement steps. We use $a_{0}^{0} \equiv a_{0,0}^{0} . \quad a_{\infty, 0}^{0}$ can be obtained from $a_{\infty, 0}^{0}=\lim _{t \rightarrow \infty}\left(a_{t, 0}^{0}+\Delta_{t, 0}^{1 \rightarrow 0}-\Delta_{t, 0}^{0 \rightarrow 1}\right)$ for high $\bar{k}$, and from Eq.(2) for low $\bar{k}$, when we only observe updates during the first iteration. This evolution is nothing but a dynamical map. The probabilities to find a configuration of neighbors allowing an update are no longer given only by $\Delta_{t, 0}^{0 \rightarrow 1}$ and $\Delta_{t, 0}^{1 \rightarrow 0}$, instead we have to count the ones constituted by a rewiring, which happens with probability $T$. That is why we can consider this kind of evolution as a dynamical map of the former process, with $a_{\infty, 0}^{0}$ as the initial population for the first rewire- 

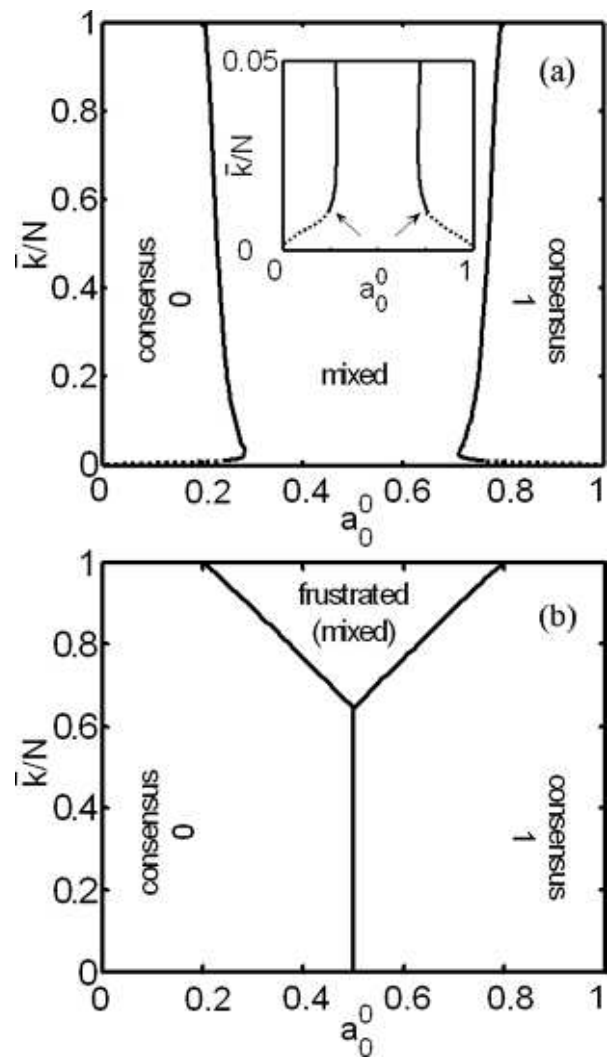

FIG. 3: Phase diagram for $a_{\infty}^{0}$ as a function of initial fraction size $a_{0}^{0}$ and connectedness, $\bar{k} / N$. Simulations where performed with ER graphs with $N=10^{3}$ and $p_{u}=0.8$. Two symmetrical regions of consensus and a mixed phase in between are observed. The dotted line indicates a smooth transition, the solid line a discontinuous one. Inset: Detail for small $\bar{k}$. Arrows mark the change from smooth to sharp transitions, positioned at $\bar{k} / N \sim 0.01$. (b) Phase diagram for $a_{\infty, \infty}^{0}$. Technically adjacency matrices with $N=10^{4}$ were generated and checked by Monte-Carlo simulations whether they allow an update at fixed $a_{0}^{0}$ and $\bar{k}$.

ment step evolving to $a_{\infty, 1}^{0}$, and so on. The transition probabilities are now given by $T \Delta_{t, \vec{t}}^{1} \vec{t}^{0}$ and $T \Delta_{t, \vec{t}}^{1 \rightarrow 0}$, since only new configurations can give rise to an update. We thus assume the master equation for a system at $T \neq 0$ after the first rewiring to be

$$
a_{\infty, \bar{t}+1}^{0}=\lim _{t \rightarrow \infty}\left(a_{t, \bar{t}}^{0}+T\left(\Delta_{t, \vec{t}}^{1 \rightarrow 0}-\Delta_{t, \vec{t}}^{0 \rightarrow 1}\right)\right) .
$$

Furthermore, one expects the existence of a critical value $k_{c}$, below which the intermediate regime (mixed state) will disappear. This will occur whenever there is no chance that a configuration of neighbors can be found leading to an update. The value for $k_{c}$ can be easily estimated: Say we have a node in state ' 1 ' and ask if an update to state ' 0 ' is possible under the given circumstances. For a given $\bar{k}$ this requires that there are at least $\left\lceil\bar{k} p_{u}\right\rceil$ neighbors in state ' 0 ' present in the set $A_{0}^{0}$. If $\bar{k}$ is above the critical value $k_{c}$ it occurs that even if all nodes

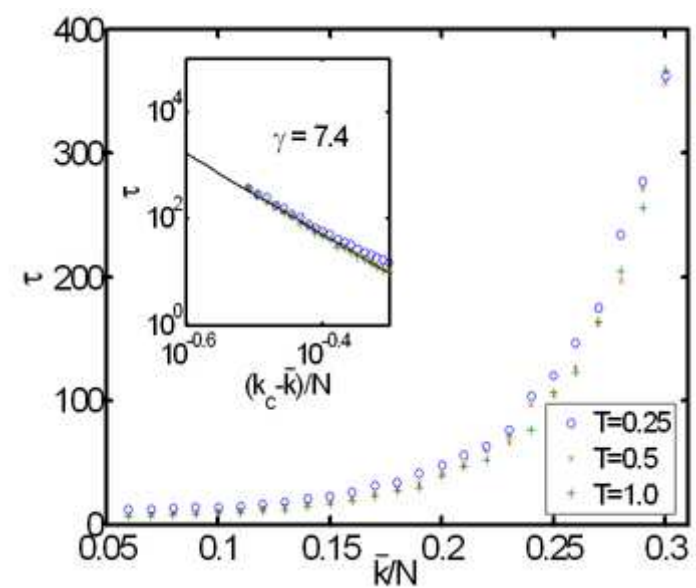

FIG. 4: Half-life time $\tau$ ( $\bar{t}$ until half the population reached consensus) vs. relative number of neighbors for $T=$ $0.25,0.5,1$. Inset: Same in $\log -\log$ scale. Scaling around the pole $k_{c} / N \sim 0.61$ with an exponent $\gamma \approx 7.4$ is suggested. $10^{3}$ initial populations with $a_{0}^{0}=0.5$ and $N=10^{2}$ were averaged.

from $A_{0}^{0}$ were neighbors of the node in state ' 1 ', there are still too many other neighboring nodes (which are then necessarily in state '1') to exceed the update threshold. This means that we can not have updates if $\left\lceil\bar{k} p_{u}\right\rceil>A_{0}^{0}$, and we get

$$
k_{c}=\frac{a_{0}^{0} N}{p_{u}} .
$$

For $p_{u}=0.8$ and $a_{0}^{0}=0.5, k_{c} \approx 0.61 N$. We next consider the time-to-convergence in the system. To this end we measure the half-life time $\tau$, of initial populations at $a_{0}^{0}=0.5$ for different connectivities $\bar{k}$, see Fig 4 The figure suggests that the observed scaling of $\tau$ could be of power-law type, with a pole at $k_{c} / N$, i.e. $\tau \propto\left(\frac{k_{c}-\bar{k}}{N}\right)^{-\gamma}$. The estimated critical exponent $\gamma \approx 7.4$ seems to be independent of temperature. Note, that the estimate is taken rather far from the pole at $k_{c}$, which suggests to interpret the actual numbers with some care.

The phase diagram for the $T \neq 0$ system is shown in Fig B(b). There are still three regimes, which are arranged in a different manner than before. Consensus is found for a much wider range of order parameters; the mixed phase is found for high connectivities, i.e. $\bar{k}>k_{c}$. The value of $k_{c}$ at $a_{0}^{0}=0.5$, as found in Fig.3(b), is 0.63 , slightly above the prediction of 0.61 . This mismatch is because we used networks with inhomogeneous degree distributions (Poisson). Whether a network allows for an update or not is solely determined by the node with the lowest degree $k$, which explains why we can still observe updates when the average degree $\bar{k}$ is near to but already above $k_{c}$. Systems in the mixed phase are frustrated. $k_{c}$ is linear in $a_{0}^{0}$ which we confirm by finding a straight line separating the frustrated drom the consensus phase, see 
Fig $3(\mathrm{~b})$. For larger $p_{u}$ the regions of consensus shrink.

\section{CONCLUSION}

Summarizing we presented a model bridging the gap between existing MR and UR models. Opinion dynamics happens on static random networks where agents adopt the opinion held by the majority of their direct neighbors only if the fraction of neighbors exceeds a pre-specified laggard-threshold, $p_{u}$. The larger this parameter the more stimulus the agent needs to adapt his opinion to the one of his direct neighborhood. This system shows two phases, full consensus and a mixed phase where opinions coexist. We studied the corresponding phase diagram as a function of the initial opinion distribution and the connectivity of the underlying networks. As the laggard-parameter $p_{u}$ increases the regions of full consensus shrink. We introduced rewiring of the underlying network during the opinion formation process and discuss the resulting consequences for the phase diagram. This social temperature introduced here differs from the usual temperature of statistical mechanics. It accounts for link fluctuations and not for the fluctuations of the state of the nodes. For $T>0$, the system can escape the frozen state $a_{\infty}^{0 / 1} \neq 1$, and global consensus can be obtained. In the case of usual temperature (opinions of nodes switch randomly) [12], a different behavior is expected. For low temperature, the system also can escape the frozen state, however for higher values of $T$ the system undergoes a transition from an ordered to an unordered phase, where $a_{\infty}=1 / 2$. Even though laggards sometimes enjoy a bad reputation as being slow and backward-oriented, so- cieties of laggards are shown to have remarkable levels of versatility as long as they are not forced to interact too much.

Supported by Austrian Science Fund FWF Projects P17621 and P19132 and COST P10 action.

* Electronic address: thurner@univie.ac.at

[1] E. Ben-Naim, L. Frachebourg, P.L. Krapivsky, Phys. Rev. E 53, 3078 (1996).

[2] P.L. Krapivsky, S. Redner, Phys. Rev. Lett. 90, 238701 (2003).

[3] M. Mobilia, S. Redner, Phys. Rev. E 68, 046106 (2003).

[4] R. Axelrod, J. Conflict. Resolut. 41, 203 (1997).

[5] P. Erdős, A. Rényi, Publ. Math. Debrecen 6, 290 (1959); Publ. Math. Inst. Hung. Acad. Sci. 5, 17 (1960).

[6] R. Albert, A.-L. Barabasi, Rev. Mod. Phys. 74, 47 (2002).

[7] D.J. Watts, S.H. Strogatz, Nature 393, 440 (1998).

[8] V. Sood, S. Redner Phys. Rev. Lett. 94, 178701 (2005).

[9] C. Castellano, V. Loretto, A. Barrat, F. Cecconi, D. Parisi, Phys. Rev. E 71, 066107 (2005).

[10] C. Castellano, D. Vilone, A. Vespignani, Europhys. Lett. 63, 153 (2006).

[11] R. Lambiotte, M. Ausloos, J.A. Holyst, Phys. Rev. E 75, 030101 (2007).

[12] R. Lambiotte, Europhys. Lett. 78, 68002 (2007).

[13] S. Galam, Physica A 274, 132 (1999).

[14] D. Stauffer, Comp. Sci. Eng. 5, 71 (2003).

[15] D. Stauffer, AIP Conf. Proc. 779, 56-68 (2005).

[16] R. Hanel, S.A. Kauffman, S. Thurner, Phys. Rev. E 72, 036117 (2005).

[17] R. Lambiotte, S. Thurner, R. Hanel, physics/0612025 (2006).

[18] D.J. Watts, Proc. Nat. Acad. Sci. 99, 5766-5771 (2002). 